\title{
The perfect smile - Part 1
}

\author{
By Rose Xi Man Poon, University of Dundee
}

Rose Xi Man Poon, a final year dental student from University of Dundee and fellow students Ai Tan, Thean Wei Yap, Christy Ng, Jonathan Lewis, Ciara Rocks, and

Erin Macaskill begin a series of articles on the topic of 'The Perfect Smile', looking at the perception of different perfect smiles around the world.

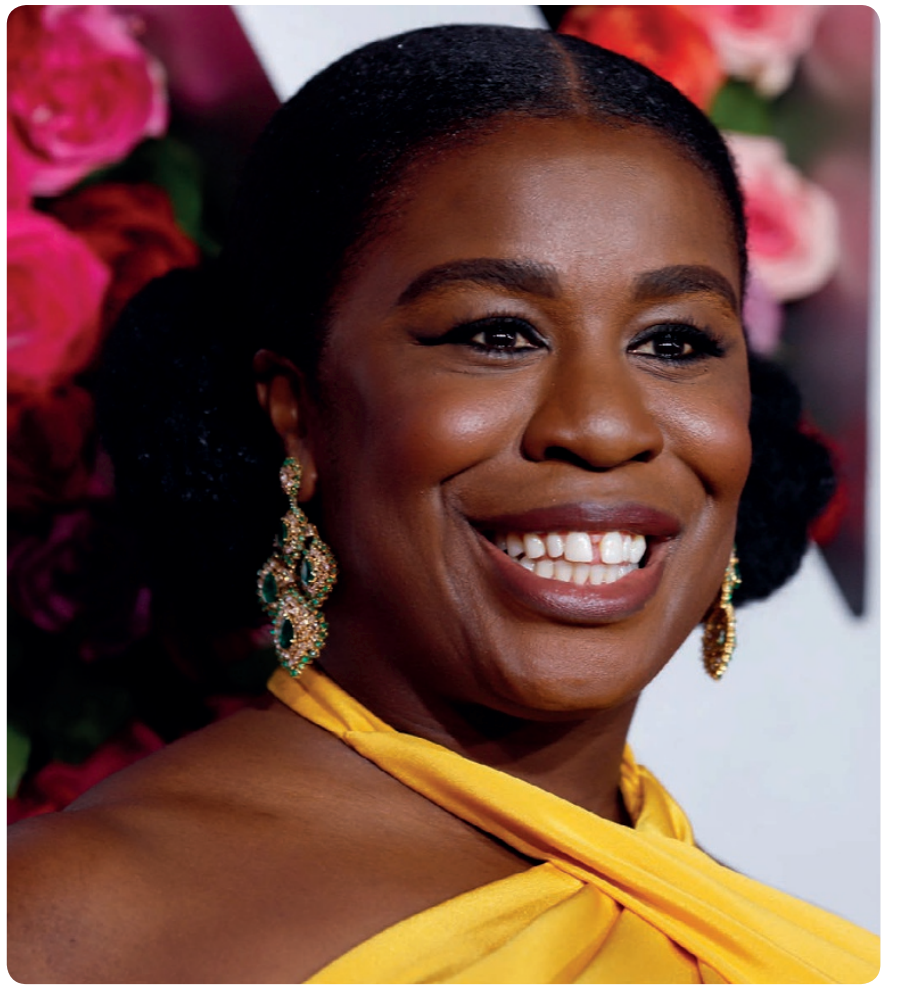

W hat makes the perfect smile? The answer to this question depends on your own idea of perfection, and can be defined differently around the world. However, in this day and age, dentistry is playing a larger role in people's lives and as a consequence there is greater desire to achieve the 'perfect smile'.

\section{AFRICA: The Beauty Removal}

In the city of Cape Town in South Africa, ritual extractions of 을 童 teenagers into adulthood. This is popularly-known as 'Passion gap' or 'Cape Flats Smile'. Fashion and peer pressure are the main reasons for this trend. Children as young as 11 have had their 产 upper front teeth removed for aesthetic purposes.

Many partial dentures are made to replace the missing teeth and have become a fashion piece of their own, by adding some decorated stones.

Removal of lower front teeth can be found in Sudan (Dinka, Nuer and Maban tribes) and mainly in the rural areas in South

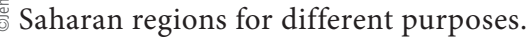

The teeth are removed quite soon after they have erupted. This act is seen as a rite of passage, and some believe that removing the lower front teeth could enhance beauty.

In Ghana, Namibia and Nigeria, a gap in women's teeth is believed by some to be a sign of beauty and fertility, says Bernice Agyekwena, a Ghanaian journalist and Gates Fellow of African Agriculture at the University of California Berkeley's Graduate School of Journalism.

'Some women even go to the extent of creating an artificial gap in their teeth because they want to meet the traditional standards set for 'African' beauty', says Agyekwena.

In some parts of Africa, sharpening or filing the teeth manually is another extreme form of body modification. Some cultures have practised this for aesthetic purpose and to distinguish the status between males and females.

Females carve their teeth into a shorter or smaller size as having long teeth is considered disrespectful towards males. The ritual of chipping off part of the upper and lower front teeth can also be seen. In Tanzania (among Makonde tribes), chipped or peg-shaped upper and lower incisors are believed to be a sign of beauty, strength or passage from teenage to adulthood.

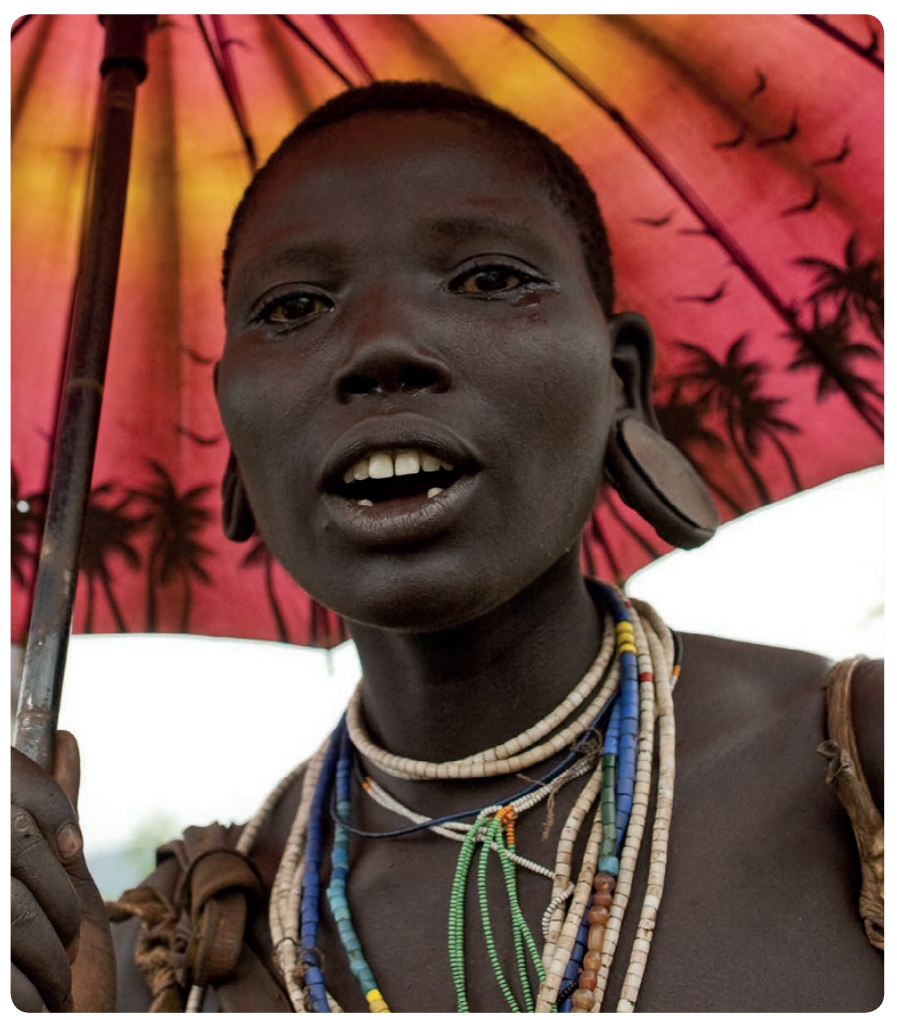

